\author{
Marquette University \\ e-Publications@Marquette
}

\title{
Characteristics of Laterally Vibrating Resonant Microcantilevers in Viscous Liquid Media
}

Russell Cox

Marquette University

Fabien Josse

Marquette University, fabien.josse@marquette.edu

Stephen M. Heinrich

Marquette University, stephen.heinrich@marquette.edu

Oliver Brand

Georgia Institute of Technology - Main Campus

Isabelle Dufour

Université de Bordeaux

Follow this and additional works at: https://epublications.marquette.edu/civengin_fac

Part of the Civil and Environmental Engineering Commons

\section{Recommended Citation}

Cox, Russell; Josse, Fabien; Heinrich, Stephen M.; Brand, Oliver; and Dufour, Isabelle, "Characteristics of Laterally Vibrating Resonant Microcantilevers in Viscous Liquid Media" (2012). Civil and Environmental Engineering Faculty Research and Publications. 67.

https://epublications.marquette.edu/civengin_fac/67 


\title{
Characteristics of laterally vibrating resonant microcantilevers in viscous liquid media
}

\author{
Russell Cox, ${ }^{1}$ Fabien Josse, ${ }^{1, a)}$ Stephen M. Heinrich, ${ }^{2}$ Oliver Brand,${ }^{3}$ and Isabelle Dufour ${ }^{4}$ \\ ${ }^{1}$ Department of Electrical and Computer Engineering, Marquette University, Milwaukee, \\ Wisconsin 53233, USA \\ ${ }^{2}$ Department of Civil and Environmental Engineering, Marquette University, Milwaukee, \\ Wisconsin 53233, USA \\ ${ }^{3}$ School of Electrical and Computer Engineering, Georgia Institute of Technology, Atlanta, \\ Georgia 30332, USA \\ ${ }^{4}$ Université de Bordeaux, CNRS, IMS Laboratory, Talence, France
}

(Received 2 November 2011; accepted 5 December 2011; published online 10 January 2012)

\begin{abstract}
The characteristics of microcantilevers vibrating laterally in viscous liquid media are investigated and compared to those of similar microcantilevers vibrating in the out-of-plane direction. The hydrodynamic loading on the vibrating beam is first determined using a numerical model. A semi-analytical expression for the hydrodynamic forces in terms of the Reynolds number and the aspect ratio (beam thickness over beam width) is obtained by introducing a correction factor to Stokes' solution for a vibrating plate of infinite area to account for the effects of the thickness. The results enable the effects of fluid damping and effective fluid mass on the resonant frequency and the quality factor $(Q)$ to be investigated as a function of both the beam's geometry and liquid medium's properties and compared to experimentally determined values given in the literature. The resonant frequency and $Q$ are found to be higher for laterally vibrating microcantilevers compared to those of similar geometry experiencing transverse (out-of-plane) vibration. Compared to transversely vibrating beams, the resonant frequency of laterally vibrating beams is shown to decrease at a slower rate (with respect to changes in viscosity) in media having higher viscosities than water. The theoretical results are compared to experimental data obtained for cantilevers completely immersed in solutions of varying aqueous percent glycerol. The increases in resonant frequency and $Q$ are expected to yield much lower limits of detection in liquid-phase chemical sensing applications. (C) 2012 American Institute of Physics. [doi:10.1063/1.3674278]
\end{abstract}

\section{INTRODUCTION}

Microcantilevers have been utilized as highly sensitive chemical sensor platforms in air. ${ }^{1-11}$ Masses in the range of picograms and femtograms have been detected using these devices, with projected detection limits on the order of attograms. ${ }^{11-13}$ While dynamically driven microcantilever chemical sensors are well suited for gas-phase detection, ${ }^{1-3,5-11,14-21}$ their usefulness as a sensing platform is limited when operating in viscous liquid media. ${ }^{7,22-29}$ Due to the additional fluid resistance (combined effects of fluidrelated inertial and viscous forces), the beam's resonant frequency, $f_{\text {res }}$, and quality factor, $Q$, will drastically decrease when the operating medium is changed from air to liquid; ${ }^{22,28,30-32}$ these decreases are due to the increases in the fluid damping and the effective fluid mass.

Several methods have been investigated to overcome this additional fluid resistance. Microcantilevers can be made stiffer by shortening their length. ${ }^{33,34}$ The resonant frequency of a microcantilever operating in a fluid is roughly proportional to the inverse of its length squared. Thus, decreasing the length by $10 \%$ would increase the resonant frequency by $\sim 23 \%$. Shorter microcantilevers also have less

\footnotetext{
a) Author to whom correspondence should be addressed. Electronic mail: fabien.josse@marquette.edu.
}

surface area when interacting with the surrounding medium, thus decreasing the amount of fluid damping and increasing the quality factor. However, the smaller surface area also decreases the amount of analyte that can be sorbed into a deposited layer when used in sensing applications. The deposition reproducibility of this sensing layer will also be less accurate for shorter beams. ${ }^{33}$

Microcantilevers can also operate in higher-order flexural modes. ${ }^{35-37}$ Higher-order modes have flexural mode shapes that have one or more points along the length of the microcantilever (besides the clamped end) that do not deflect as a function of time. ${ }^{36}$ Both theoretical ${ }^{36}$ and experimental investigations ${ }^{35,37}$ show that, when working in air or liquids, the quality factor of a microcantilever operating in a higherorder mode is higher than that of the same microcantilever operating in the fundamental mode. However, there are also some drawbacks to operating in higher-order flexural modes, such as an increase in support loss.

When working in a vacuum, experiments have shown that the quality factor of the microcantilever decreases with an increasing mode number. ${ }^{38}$ The support losses for a particular microcantilever operating in the second mode have been shown to be 10 times larger than operating in the first mode. ${ }^{33,39}$ This tends to be less of a concern when operating in air or liquid, since the viscous losses generally dominate the support losses. ${ }^{34}$ Moreover, it is also relatively more 


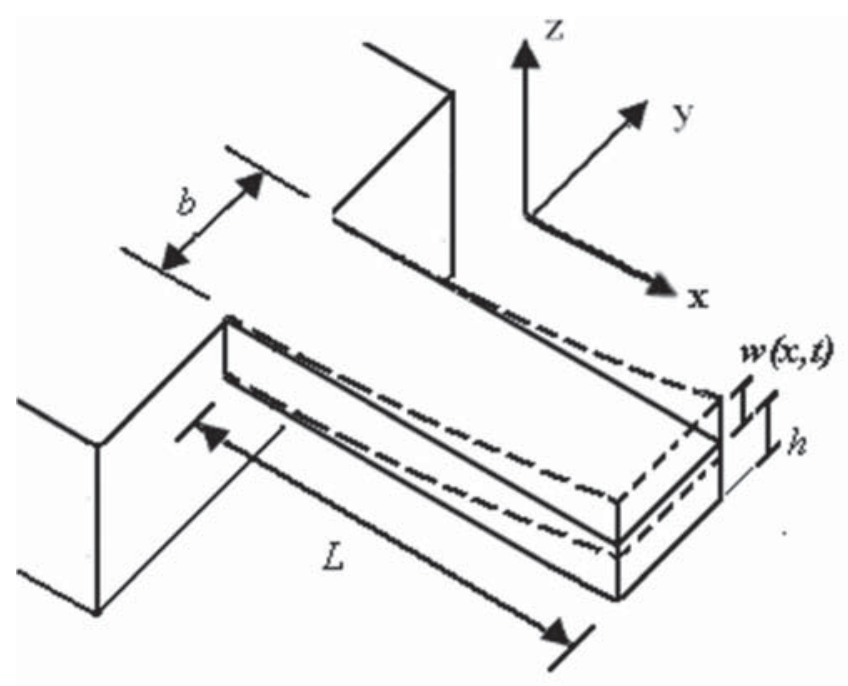

Out of plane vibration

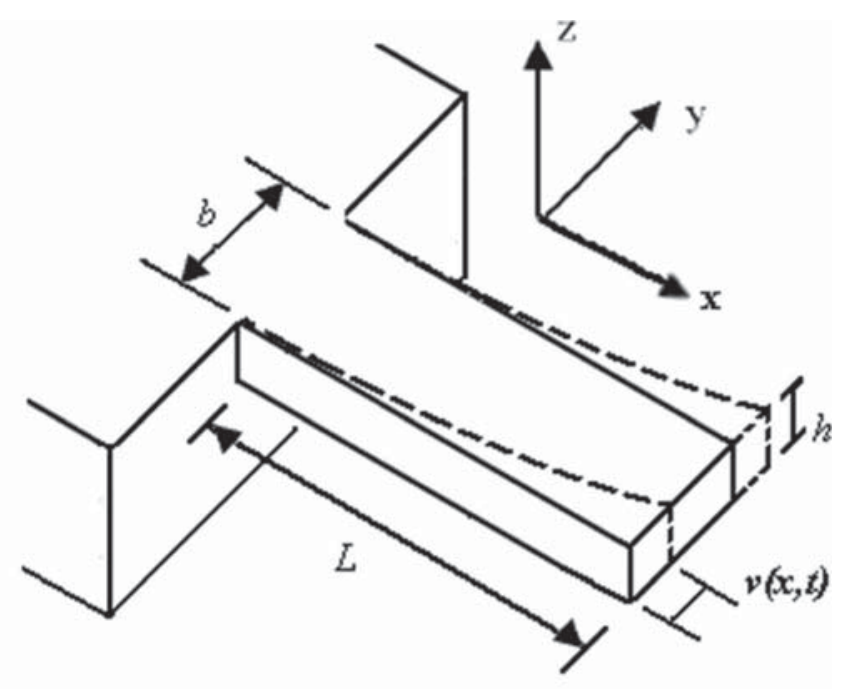

In-plane vibration

FIG. 1. Geometry of a microcantilever of length $L$, width $b$, and thickness $h$ vibrating in the out-of-plane direction (left) with a deflection of $w(\mathrm{x}, \mathrm{t})$ and vibrating in the in-plane direction (right) with a deflection of $v(\mathrm{x}, \mathrm{t})$.

difficult to excite these higher-order modes to achieve a sufficiently strong signal. ${ }^{38}$

Another promising method for overcoming the additional fluid resistance is by exciting the microcantilever in an unconventional vibration mode. Dynamically driven microcantilevers are commonly excited flexurally in the transverse or out-of-plane direction, as indicated in Fig. 1. 1,3,7,26,32,40-46 Another vibration mode that has been previously investigated in the literature is the in-plane (lateral) flexural mode. ${ }^{22,35,38,47-51}$ This reduces the amount of fluid resistance by decreasing the amount of fluid drag on the leading edge of the beam. The reduction in the fluid resistance results in an increase in the resonant frequency and the quality factor of the microcantilever compared to similar beams vibrating transversely. ${ }^{22,30,38}$ In many cases, the increase in these characteristics can be investigated using standard beam theory, if the hydrodynamic forces acting on the beam are properly modeled.

There have been several attempts to model the hydrodynamic forces acting on a beam vibrating in the in-plane direction. $^{22,38,49,50,52,53}$ While a well-known expression for the hydrodynamic forces acting on a beam undergoing transverse vibration has been given in the literature, ${ }^{23}$ this expression cannot be used to find the hydrodynamic forces acting on a microcantilever vibrating in the in-plane direction as it neglects the effects of fluid on the larger faces (of dimension $b$ ) of the laterally vibrating beam. A more accurate approximation of the total hydrodynamic force acting on a laterally vibrating microcantilever in liquid should include both the effects of the pressure and shear stress exerted by the fluid on all faces of the beam. Due to the symmetry of the problem, the hydrodynamic forces acting in the vertical direction on a beam with a rectangular cross-section vibrating laterally will cancel each other out. Only the hydrodynamic forces acting in the horizontal direction will then affect the characteristics of the beam. These forces are the fluid shear force acting on the large faces and the pressure force acting on the small faces (of dimension $h$, see Fig. 2). It can be assumed that the microcantilever is long enough so that the hydrodynamic force acting on the surface at the free end of the beam is negligible.

When the beam's thickness is small compared to its width, the beam can also be approximated as a ribbon. ${ }^{54,55}$ (This has previously been shown valid for beams vibrating transversally. ${ }^{23,31}$ ) In 1851, Stokes investigated the forces acting on an infinitely wide flat plate brought from rest to sinusoidal, in-plane vibration. ${ }^{56}$ This is commonly called Stokes' second problem. ${ }^{57}$ The solution for a laterally vibrating beam of infinitely thin cross-section can be reduced to the steady-state solution of Stokes' second problem if the beam under investigation is also quite wide relative to the boundary layer thickness of the fluid. ${ }^{31}$ Recently, this Stokes-type fluid resistance has been used to obtain analytical results for the quality factor associated with the viscous

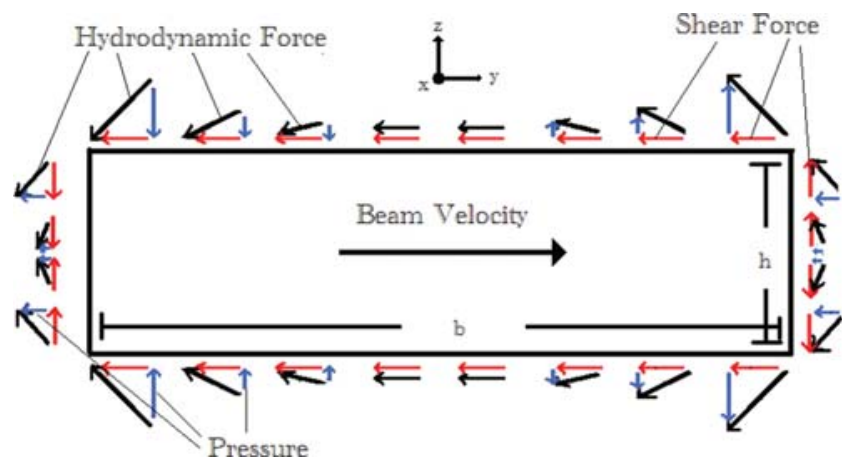

FIG. 2. (Color online) The hydrodynamic force acting on a laterally vibrating beam can be conceptually broken up into the forces acting parallel to the surface (the fluidic shear force) and forces acting perpendicular to the surface (the pressure force). 
fluid losses for laterally vibrating beams in liquid. ${ }^{49,50}$ The quality factors predicted using this model were found to well agree with the experimentally determined quality factors of laterally excited beams when $b \gg h$. However, this method neglected edge effects, which could be significant for beams whose width $b$ is small. Furthermore, it also neglects the fluid resistance due to pressure on the thin faces of the beam (those of corresponding dimension $h$ ), which may be important for many practical beam geometries.

The hydrodynamic function of a transversely vibrating beam of ribbon-like cross-section was previously found in Ref. 52 by use of the method of moments to solve for the velocity of the fluid around the ribbon. Recently, an investigation expanded upon this work to obtain numerical results for both the transverse and lateral hydrodynamic function that accounted for both the beam's aspect ratio $(h / b)$ and the Reynolds number ( $\mathrm{Re}$ ) of the fluid flow. ${ }^{47}$ While the numerical results in the present paper match closely with those given in Ref. 47, the previous work only provides values of the hydrodynamic function at specific discrete combinations of aspect ratio and Reynolds number. In the present paper, an analytical expression will be given for this function that can be used over wide, continuous ranges of $h / b$ and Re. A comparison between the results of Ref. 47 and those of the present investigation will be provided. Other investigators have attempted to use finite element analysis (FEA) in order to account for the effects of thickness. ${ }^{31,58}$ Like the numerical method used in Ref. 47, FEA allows for the pressure and shear force to be calculated on the beam's cross-section, as well as in the medium surrounding the beam. It is noted, however, that Refs. 31 and 58 only investigate transversely vibrating beams.

In this article, an FEA model will be developed and utilized to calculate the hydrodynamic forces acting on a laterally vibrating beam. This method will account for both the edge effects and thickness effects, which are not accounted for when assuming Stokes-type fluid resistance. A set of correction factors will then be obtained so that the Stokes hydrodynamic function may be mapped into one that accounts for these additional effects. Using the resulting hydrodynamic function, relevant beam characteristics, such as the resonant frequency and quality factor, will be obtained and investigated as functions of beam geometry and medium properties, including density and dynamic viscosity. The advantages of operating in the in-plane flexural mode compared to the out-of-plane flexural mode will also be investigated. The results will be utilized to identify microcantilever geometries that will yield more desirable characteristics for use in sensing applications than those provided by conventional (out-of-plane) microcantilever devices.

\section{GENERAL THEORY}

In a vacuum, using standard Euler-Bernoulli beam theory, the equation of motion for a laterally vibrating microcantilever is

$$
E I_{\text {lat }} \frac{\partial^{4} v(x, t)}{\partial x^{4}}+\rho_{B} b h \frac{\partial^{2} v(x, t)}{\partial t^{2}}=F_{y}(x) e^{j \omega t},
$$

where

$$
I_{l a t}=b^{3} h / 12 \text {. }
$$

In Eq. (1), $E$ and $\rho_{B}$ are the Young's modulus and mass density of the beam material, respectively, $v(x, t)$ is the in-plane (or lateral) deflection of the beam, and $F_{\mathrm{y}}(x)$ is the positiondependent amplitude of the forcing function per unit length operating at an angular frequency of $\omega$. Note that the equation for the moment of inertia, $I_{\text {lat }}$, has the width cubed instead of the thickness (as is the case in transverse vibration). The same Young's modulus is assumed for both lateral and transverse vibration, i.e., that corresponding to axial stress/strain along the longitudinal direction of the cantilever. Thus, the flexural rigidity $(E I)$ of a beam undergoing lateral vibration is a factor of $(b / h)^{2}$ larger than that of the same beam undergoing transverse vibration. This indicates that laterally vibrating beams (compared to transversely vibrating beams) will be stiffer and have higher resonant frequencies. In a vacuum, this represents the only difference between lateral and transverse vibration when solving the equation of motion.

Euler-Bernoulli beam theory, as employed herein, places several assumptions on the beam geometry and deflection:

- The beam's cross-sectional area, density, and Young's modulus are uniform over the length of the beam

- The length of the beam greatly exceeds its width

- The amplitude of the vibration of the beam is sufficiently small, so that the slope of the deflected beam is much smaller than unity; this permits the beam curvature to be approximated by $\partial^{2} v(x, t) / \partial x^{2}$

These assumptions generally hold true for standard commercially available microcantilevers. ${ }^{23,38}$ However, some of the geometries investigated in this work do not have lengths that greatly exceed their widths. The effects of violating this assumption will be discussed.

When a microcantilever is excited in a viscous liquid medium, an additional force from the medium affects the microcantilever and the equation of motion is modified to

$$
E I_{l a t} \frac{\partial^{4} v(x, t)}{\partial x^{4}}+\rho_{B} b h \frac{\partial^{2} v(x, t)}{\partial t^{2}}=F_{y}(x) e^{j \omega t}+F_{m d, l a t}(x, t) .
$$

This additional hydrodynamic force, $F_{m d, l a t}$, is a force per unit length that is partially out-of-phase with the displacement and can be represented as

$$
F_{m d, l a t}(x, t)=-g_{1, l a t} \frac{\partial v(x, t)}{\partial t}-g_{2, l a t} \frac{\partial^{2} v(x, t)}{\partial t^{2}},
$$

where $g_{1, \text { lat }}$ and $g_{2, \text { lat }}$ are time-independent coefficients associated with the fluidic damping force per unit length and the fluidic inertial force (effective fluidic mass) per unit length, respectively. ${ }^{21,22,31}$ It is common to normalize $F_{m d, l a t}$ into a dimensionless form called the hydrodynamic function, $\Gamma_{\text {lat }}$, where 


$$
\begin{aligned}
g_{1, l a t} & =\frac{\pi}{4} \rho_{L} b^{2} \Gamma_{l a t, I}(\operatorname{Re}, h / b) \omega, \\
g_{2, l a t} & =\frac{\pi}{4} \rho_{L} b^{2} \Gamma_{l a t, R}(\operatorname{Re}, h / b),
\end{aligned}
$$

where the Reynolds number of the system, Re, is defined in terms of $\rho_{L}$ and $\eta$, which are the mass density and dynamic viscosity of the fluid, respectively.

The hydrodynamic function, $\Gamma_{l a t}$, is the total hydrodynamic force per unit length normalized to the amount of force per unit length it would take to excite fluid occupying a circular cylindrical volume with a diameter equal to the microcantilever's width to the same velocity as the microcantilever. ${ }^{25,53}$ The subscripts $R$ and $I$ in Eqs. (3) and (4) represent the real and imaginary parts of the hydrodynamic function, respectively, where $h / b$ is the aspect ratio of the beam cross-section. The Reynolds number is a measure of the relative size of the fluid's inertial and viscous forces. An analytical expression for the Reynolds number can be defined from the ratio of the inertial term to the viscous term in the equation of motion of the fluid. The linearized incompressible form of the Navier-Stokes' equation can be used to model the fluid if the fluid is assumed incompressible and the velocity gradient of the fluid is small. The fluid can be considered incompressible as the wavelength of the microcantilever's vibration in the cases of interest in this work greatly exceeds the width of the microcantilever, which is the dominant length scale in the flow. ${ }^{23}$ The velocity of the fluid is related to the velocity of the vibrating microcantilever. Assuming that the microcantilever's amplitude of vibration is far smaller than any length scale in the microcantilever's geometry, the velocity gradient of the beam and fluid can be considered small. Thus, the equation of motion for the fluid can be given as $^{31,49,59}$

$$
\rho_{L} \frac{\partial u}{\partial t}=-\nabla P+\eta \nabla^{2} u
$$

where $P$ and $u$ are the pressure and velocity at a particular point in the fluid, respectively. The term $\rho_{L} \frac{\partial u}{\partial t}$ is the term related to the fluid's inertial forces, while $\eta \nabla^{2} u$ is the term related to the fluid's viscous forces. The Reynolds number, in this form sometimes called the non-dimensional frequency ${ }^{52}$ or one fourth of the Valensi number, ${ }^{60}$ can then be calculated by simplifying the ratio of these terms as $\operatorname{Re}=\rho_{L} \omega \mathrm{b}^{2} /(4 \eta)$. The viscosity is multiplied by a factor of four to keep the definition consistent with the one given in Ref. 52.

The properties of the medium of operation, along with the excitation frequency $\omega$, will determine the skin depth of the liquid layer surrounding the vibrating beam. The skin depth or boundary layer thickness, denoted $\delta$, is defined as the distance over which the fluid velocity decays to $1 / e$ of its maximum value. ${ }^{61}$ The larger the skin depth, the larger the amount of fluid excited by the vibrating beam. Physically, the boundary layer thickness can be thought of as the amount of fluid trapped in the vortex created by the vibrating microcantilever. $^{62}$ The boundary layer thickness can be given as $\delta=\left(2 \eta / \rho_{L} \omega\right)^{1 / 2}$, which can be rewritten in terms of the Reynolds number as

$$
\delta=\frac{b}{\sqrt{2 \mathrm{Re}}} .
$$

The hydrodynamic function for a transversely vibrating beam was found to be a function of $\delta / b$ or a function of the inverse of the square-root of the Reynolds number. $^{31,61}$ It is expected that the hydrodynamic function for a laterally vibrating beam will also depend on this ratio and, thus, on the Reynolds number. When $\delta / b$ and $h / b$ are sufficiently small, the hydrodynamic function for the lateral case can be obtained from the steady state solution of Stokes' second problem. For this special case, the shear force per unit area from the fluid, $\tau$, acting on the top of the beam is ${ }^{56}$

$$
\tau=-V_{0} e^{j \omega t} \sqrt{\frac{\eta \rho_{L} \omega}{2}}(1+j)
$$

where $V_{0}$ is the amplitude of the velocity at a particular point along the beam. Due to the symmetry of the problem, the total shear force is twice that on the top of the beam. Assuming that the shear force is independent of $y$, the total shear force (per unit length) over a beam of width $b$ is given by

$$
F_{m d, l a t}=2 \int_{-b / 2}^{b / 2} \tau d y=-2 V_{0} e^{j \omega t} \sqrt{\frac{\eta \rho_{L} \omega}{2}}(1+j) b .
$$

The above equation states that the real and imaginary part of the hydrodynamic force are equal in magnitude and are proportional to the width $b$ and the velocity amplitude $V_{0}$ and are also dependent on the square-root of the product of the viscosity, fluid mass density, and excitation frequency. The hydrodynamic function is then given as

$$
\Gamma_{\text {lat }, \text { Stokes }}(\operatorname{Re})=\frac{4}{\pi \sqrt{2 \operatorname{Re}}}(1+j)=\frac{4 \delta}{\pi b}(1+j) .
$$

It is noted that this solution does not account for the edge effects when $\delta / b$ is not sufficiently small; nor does it account for the pressure effects on the faces of dimension $h$. Using finite element analysis (FEA), a set of correction factors can be derived and applied to Eq. (9) to account for both the edge and pressure effects.

\section{NUMERICAL SIMULATION}

A model of the laterally vibrating beam's cross-section in fluid was defined using the FEA program ANSYS. A 2D mesh representing the cross-section of the fluid surrounding an oscillating beam was modeled, with an unmeshed rectangle representing the beam's cross-section. Thus, the beam's cross-section is assumed to not undergo deformation. A square outer boundary of the fluid was assumed, with the beam cross-section having a constant width and a variable thickness. Three boundary conditions were placed on the mesh. A lateral sinusoidal velocity was imposed on the cross-section, and a zero displacement and zero pressure boundary condition were placed on the outer boundary. The mesh was defined so that the node density was higher near the beam and lower near the outer boundary. The outer 
boundary was set far enough away from the vibrating beam (roughly 44 times the largest skin depth) so that its effects could be neglected. The mass density and dynamic viscosity of the liquid were set to that of water $\left(\rho_{\mathrm{L}}=1000 \mathrm{~kg} / \mathrm{m}^{3}\right.$ and $\eta=1 \mathrm{cP})$. The amplitude of the displacement was held constant while the beam's frequency and velocity were varied in order to investigate the effects of operating in different Reynolds numbers. A transient analysis was performed over 2 cycles, which was determined (by evaluating the amplitude variation over the two cycles) to be long enough for the transient effects to become negligible for the frequency range considered. The pressure and shear force (per unit length) acting on the fluid-beam interface in the lateral direction were then computed at 200 different times per cycle over two cycles. The amplitude of the hydrodynamic force was then determined. Using the amplitude, the average phase offset between the hydrodynamic force and the imposed displacement over the last period was determined. The hydrodynamic function over a range of aspect ratios $(h / b)$ and Reynolds numbers could then be calculated from the determined hydrodynamic force.

In order to determine whether the mesh yielded convergent results for the ranges of Reynolds number (from 10 to 10000 ) and aspect ratio (from 1/56 to 1 ) investigated, the hydrodynamic function was computed as the mesh density was varied. The range of Reynolds numbers chosen corresponds to the common range of Reynolds numbers when using laterally vibrating microcantilevers with practical geometries. The aspect ratio initially ranged from 1 to $1 / 100$, with equal steps on a logarithmic scale in order to investigate thin beams more thoroughly. However, the smallest aspect ratio investigated was modified to $h / b=1 / 56$, as $h / b=1 / 100$ proved to require an impractically high mesh density for the chosen range of Reynolds numbers in order to produce a convergent force. The number of elements used was varied from about 1000 to approximately 80000 , and the hydrodynamic force amplitude and phase were observed to converge to particular values. When increasing the number of elements of the convergent mesh chosen by $\sim 70 \%$, the largest percentage difference in the hydrodynamic function for the aspect ratios and Reynolds numbers investigated was $5.19 \%$ for the real part of the hydrodynamic function and $2.87 \%$ for the imaginary part of the hydrodynamic function. The largest differences were noted for microcantilevers with aspect ratios of $\sim 1 / 56$. This is expected, as thinner microcantilevers require a higher number of elements to accurately model the forces along the thickness. Increasing the number of elements used in the chosen mesh would result in a slightly more accurate solution for the hydrodynamic function, but at an extreme cost of time and computational complexity.

The same method was also used to compute the hydrodynamic forces acting on a transversely vibrating beam, and the results were compared to those given in the literature. ${ }^{23}$ The real part of the hydrodynamic function calculated using the chosen mesh differs from the values calculated using the method given in Ref. 23, ranging from $1.6 \%$ lower when $\operatorname{Re}=10$ to $4.3 \%$ higher when $\operatorname{Re}=10000$. The imaginary part of the hydrodynamic function also differs from the values given in Ref. 23, ranging from 5.4\% lower when $\operatorname{Re}=10$ to $10.3 \%$ lower when $\operatorname{Re}=3162$. This difference is most likely due to the assumption of zero thickness made in the literature, such as in Ref. 23. It is noted that the recent work done in Ref. 47, which includes the effects of a finite aspect ratio, also indicates that the hydrodynamic function of a transversely vibrating beam of finite thickness differs from that of a transversely vibrating ribbon. There could also be some difference due to approximations associated with the numerical modeling. However, the differences in the two hydrodynamic functions are not large enough to cause significant differences in the predicted characteristics of beams vibrating in the fluid.

Figures 3(a) and 3(b) show the numerical results of the hydrodynamic function as a function of the Reynolds number and aspect ratio (on a log scale) for the real and imaginary part of the hydrodynamic function, respectively, of a laterally vibrating beam. As the thickness of the beam decreases, the hydrodynamic function tends to that of an infinitely thin beam. When $\operatorname{Re} \gg 1$ and $h / b \ll 1$, Stokes' solution for a ribbon (Eq. (9)) can be used to estimate the hydrodynamic function. For instance, there is less than a $1 \%$ difference between Stokes' theory and the real part of the hydrodynamic function predicted for $\mathrm{Re}=10000$ and $h / b=1 / 56$. However, the imaginary part of the hydrodynamic function predicted for that configuration has a $14.3 \%$ difference with Stokes' theory, which is the smallest difference over the range of Reynolds numbers and aspect ratios investigated. If the results for $h / b=1 / 56$ are excluded, the minimum differences between Stokes' theory and the predicted real part and imaginary part of the hydrodynamic function are both $19 \%$ over the range of Reynolds numbers and aspect ratios investigated. This is due to Stokes' solution neglecting both the edge effects and thickness effects of the beam and also errors in the numerical simulations. It is again recalled that, for the comparison, the smallest aspect ratio investigated was $h / b=1 / 56$, as $h / b=1 / 100$ proved to require an impractically high mesh density for the chosen range of Reynolds numbers in order to produce a convergent force.

Figure 4 shows the real and imaginary hydrodynamic function for a beam with $h / b=1 / 56$ over a range of Reynolds numbers. Also plotted is the hydrodynamic function calculated using Stokes' solution and the values for the hydrodynamic function given by Ref. 47 for a laterally vibrating ribbon, accounting for the edge effects. Note that, in Fig. 4, the discrepancy for low Reynolds numbers is mainly due to the edge effects. Accounting for the edge effects, the remaining discrepancy could be due to neglecting the effects of thickness.

It can be seen from Fig. 3(b) that both the imaginary part of the hydrodynamic function and Stokes' solution converge to zero as the Reynolds number is increased. However, the real part of the hydrodynamic function, as shown in Fig. 3(a), does not follow this trend. Even in an inviscid medium (or Re approaching infinity), a laterally vibrating beam of finite thickness will still displace fluid mass. An approximation for the hydrodynamic function for the case of an inviscid medium is given ${ }^{47}$ as 

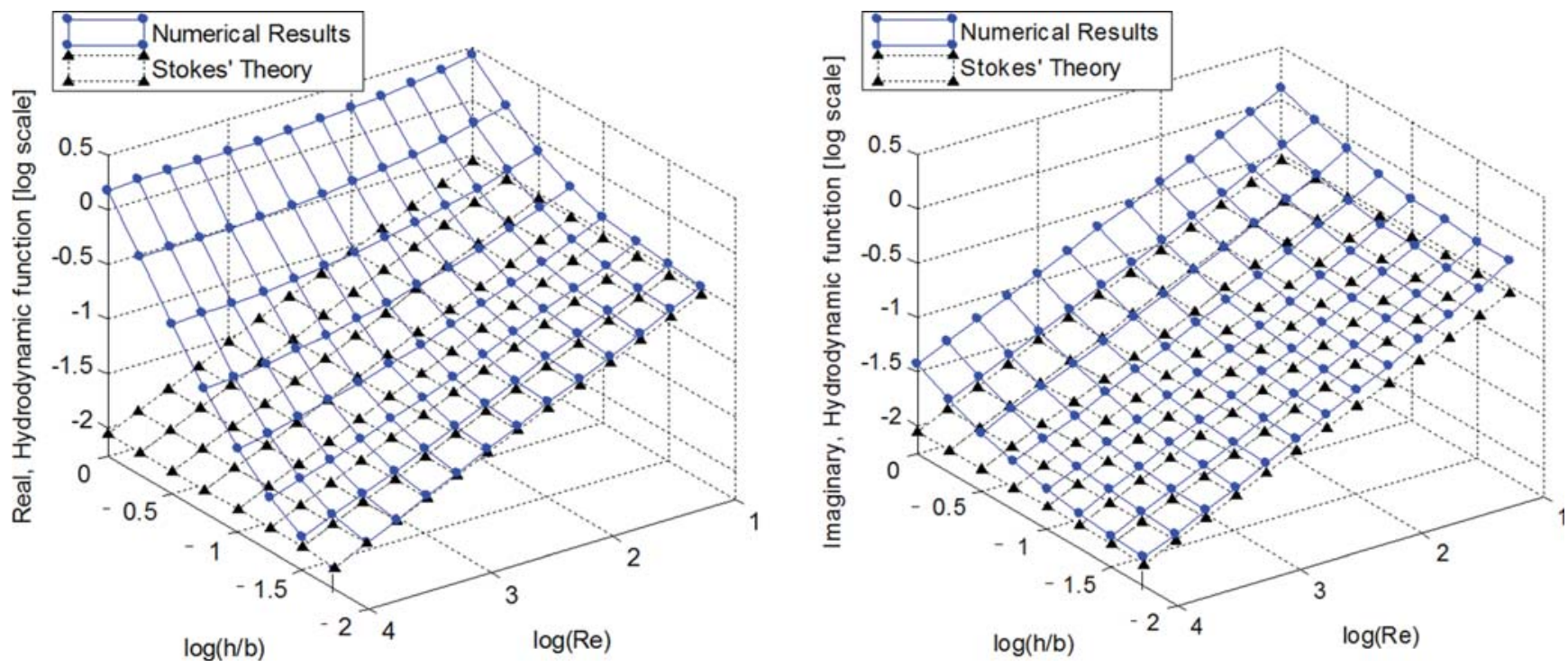

FIG. 3. (Color online) (a) and (b): The real (left) and imaginary (right) parts of the hydrodynamic function as a function of the aspect ratio and the Reynolds number calculated using a finite element model and compared to the thickness-independent analytical results calculated using Stokes' theory. The ratio of these two produces a correction factor that may be applied to Stokes' solution to account for the effects of thickness.

$$
\Gamma_{\text {lat,inviscid }}(h / b)=\frac{2}{\pi^{2}}\left(\frac{h}{b}\right)^{2}\left(1+2 \ln \left(\frac{4 \pi b}{h}\right)\right), \quad\left(\frac{h}{b}\right) \ll 1 \text {. }
$$

Note that Eq. (10) has no imaginary part, as there is no viscous damping on a beam operating in an inviscid medium. Figure 5 shows the numerical results for the real and imaginary part of the hydrodynamic function for an aspect ratio of 1/10, varying the Reynolds number from 10 to 10 000. Also plotted in Fig. 5 are Stokes'solution and the approximation

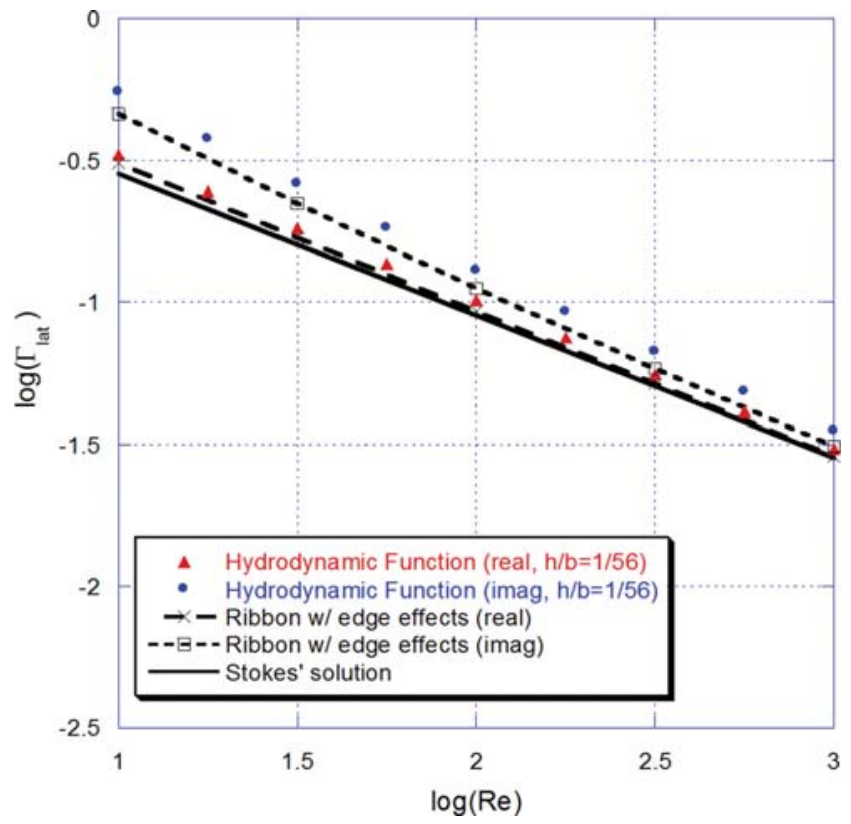

FIG. 4. (Color online) The real and imaginary part of the hydrodynamic function of a laterally vibrating beam as a function of the Reynolds number calculated using a finite element model, assuming an aspect ratio of $h / b=1 / 56$, compared to the thickness-independent analytical results calculated using Stokes' theory. for the hydrodynamic function of an inviscid medium given by Eq. (10). While the imaginary part of the hydrodynamic function approaches (albeit rather slowly) Stokes' solution as the Reynolds number increases, the real part approaches the approximation given by Eq. (10).

The results shown in Figs. 3(a) and 3(b) are similar to the results given in Ref. 47. Direct comparison is limited to aspect ratios of $h / b=1$ and $1 / 10$, as different step sizes were used when varying the aspect ratio. If an analytical expression for the hydrodynamic function that covered all the

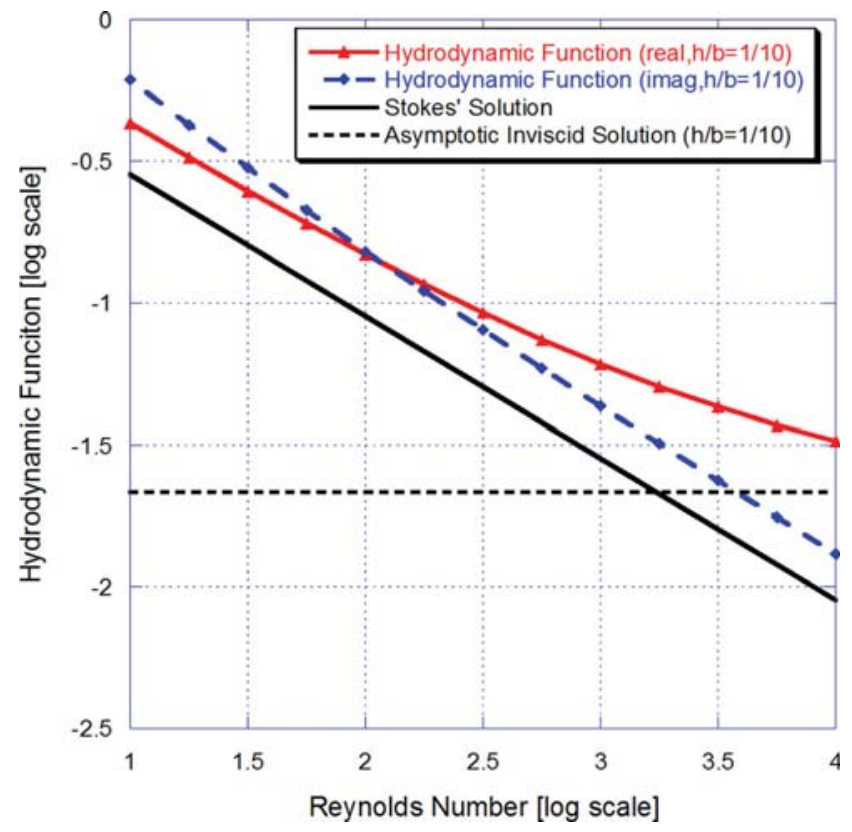

FIG. 5. (Color online) The real and imaginary parts of the hydrodynamic function as a function of the Reynolds number calculated using a finite element model and assuming an aspect ratio of $1 / 10$ compared to the thickness-independent analytical results calculated using Stokes' theory and the Reynolds number-independent asymptotic inviscid solution. 
combinations of aspect ratios and Reynolds numbers used in this investigation could be obtained, the two techniques could be more thoroughly compared. More importantly, one could rapidly obtain the hydrodynamic function for any arbitrary aspect ratio and Reynolds number within the ranges investigated. Such an expression will be pursued in what follows.

An analytical form of the hydrodynamic function can be obtained using both Stokes' solution and the numerical results. The ratios of the numerical results and the values of the hydrodynamic function calculated from Stokes' theory are used to obtain a correction factor for both the thickness and the edge effects for the real and imaginary parts of the hydrodynamic function. Fitting the trends in the ratio and assuming that the correction factor goes to one for high Reynolds numbers and thin beams, the hydrodynamic function with edge and thickness effects taken into account is given by

$$
\Gamma_{\text {lat }}(\operatorname{Re}, h / b)=\frac{4}{\pi \sqrt{2 \operatorname{Re}}}\left(C_{R}+j C_{I}\right),
$$

where the correction factors for the real and imaginary parts of the hydrodynamic function are given, respectively, by

$$
\begin{gathered}
C_{R}=\left(1.658\left(\frac{h}{b}\right)^{1.83} \sqrt{\operatorname{Re}}+3.08\left(\frac{h}{b}\right)^{0.85}+1\right), \\
C_{I}=\left(\left(2.56-1.321\left(\frac{h}{b}\right)\right) \frac{1}{\sqrt{\operatorname{Re}}}+3.108\left(\frac{h}{b}\right)^{0.85}+1\right) .
\end{gathered}
$$

The imaginary part of Eq. (11) is within $5.7 \%$ of the results from the numerical simulations over the investigated ranges of aspect ratio $(1 / 56 \leq h / b \leq 1)$ and Reynolds number $(10 \leq \operatorname{Re} \leq 10000)$. For the same range, the real part of Eq. (11) is within $20.5 \%$ of the numerical results. It is noted that this large discrepancy for the real part occurs when the Reynolds number is high. When $\operatorname{Re}<1000$, the discrepancy between the real part of the expression and the numerical results decreases to no more than $5.8 \%$. A more complicated fitting model and, hence, a more complicated correction factor could be used to improve the accuracy of the semianalytical method; however, at a high Reynolds number, the microcantilever's mass is usually much larger than the effective fluid mass, in which case the error in the real part of the hydrodynamic function will have minimal effect on the microcantilever's dynamic response.

Using this analytical expression, the hydrodynamic function can be calculated for aspect ratios and Reynolds numbers between the simulated data points, and the results could be used to rapidly evaluate the characteristics of a laterally vibrating microcantilever in a viscous liquid medium. The real and imaginary parts of the hydrodynamic function can also be compared to results given recently in the literature. ${ }^{47}$ The difference between the two techniques, using the results in Ref. 47 as a reference, ranges from $-1.82 \%$ to $5.88 \%$ for the real part of the hydrodynamic function and from $-9.85 \%$ to $2.86 \%$ for the imaginary part of the hydrodynamic function. The largest difference occurs when using an aspect ratio of $1 / 50$ and a Reynolds number of 10 . The difference in the hydrodynamic function is larger when the Reynolds number is low. Using only the results for $\mathrm{Re} \geq 100$, the range decreases from $-1.69 \%$ to $3.03 \%$ for the real part and from $-4.61 \%$ to $1.56 \%$ for the imaginary parts of the hydrodynamic function. The methods used in this work and in Ref. 47, while different, still yield similar values for the hydrodynamic function. However, as indicated earlier, the obtained analytical expression can allow a more rapid evaluation of the microcantilever characteristics when operating in the liquid-phase.

\section{RESULTS AND DISCUSSION}

\section{A. Frequency spectrum}

Assuming a sinusoidal driving force, the steady-state deflection of the beam as a function of the position along its length can be derived from the equation of motion, ${ }^{32}$ Eq. (2), as

$$
v(x, t)=\sum_{i=1}^{\infty}\left(\frac{1}{E I_{\text {lat }} \beta_{i}^{4}-\left(\rho_{B} b h+g_{2, \text { lat }}\right) \omega^{2}+j g_{1, \text { lat }} \omega} \frac{\int_{0}^{L} F_{y}(x) \phi_{i}(x) d x}{\int_{0}^{L} \phi_{i}^{2}(x) d x}\right) \phi_{i}(x) e^{j \omega t},
$$

where $\phi_{i}(\mathrm{x})$ is the $i$ th flexural mode shape of an elastic beam (in vacuum), defined as

$$
\phi_{i}(x)=\left(\frac{\left(\cos \beta_{i} x-\cosh \beta_{i} x\right)\left(\cos \beta_{i} L+\cosh \beta_{i} L\right)+\left(\sin \beta_{i} x-\sinh \beta_{i} x\right)\left(\sin \beta_{i} L-\sinh \beta_{i} L\right)}{\left(\sin \beta_{i} L-\sinh \beta_{i} L\right)}\right),
$$

and the mode number $\beta_{i}$ is the $i$ th root of $\left(\cos \beta_{i} L \cosh \beta_{i} L\right)+1=0$. For $i=1, \beta_{1} L \approx 1.8751$. Investigating only the amplitude of the deflection of the beam at the tip, one has

$$
v_{\max }(L)=\sum_{i=1}^{\infty}\left(\frac{1}{\sqrt{\left(E I_{\text {lat }} \beta_{i}^{4}-\left(\rho_{B} b h+g_{2, \text { lat }}\right) \omega^{2}\right)^{2}+\left(g_{1, l a t} \omega\right)^{2}}} \frac{\int_{0}^{L} F_{y}(x) \phi_{i}(x) d x}{\int_{0}^{L} \phi_{i}^{2}(x) d x}\right) \phi_{i}(L) .
$$


Thus, for a given load function $F_{y}(x)$, the amplitude of the tip deflection as a function of the excitation frequency in water can then be calculated by using the correct values for $g_{1, \text { lat }}$ and $g_{2, \text { lat }}$ using Eqs. (3) and (4) and the hydrodynamic function calculated using Eq. (11). It is shown in Fig. 6 that, simulating a laterally excited microcantilever with a geometry of $200 \times 45 \times 12 \mu \mathrm{m}^{3}$ with an assumed Young's modulus of silicon along the $<110>$ direction $(169 \mathrm{GPa})^{63}$ and a concentrated harmonically varying force at the tip, the resonant frequency and 3-dB bandwidth are higher for the first inplane flexural mode compared to the first out-of-plane flexural mode. The resonant frequency of a beam vibrating in the in-plane direction (when $b>h$ ) is larger than the resonant frequency of a beam with the same geometry vibrating in the out-of-plane direction, due to its increased stiffness.

\section{B. Resonant frequency}

The derivation of the formulae for the resonant frequency and the quality factor for a dynamically driven microcantilever vibrating in the in-plane direction in a viscous liquid medium follows the same procedures as those in Ref. 32, except for the use of different values of the moment of inertia $\left(I_{\text {lat }}\right)$ and the hydrodynamic force (i.e., $g_{1, \text { lat }}$ and $\left.g_{2, \text { lat }}\right)$. Utilizing Eq. (14), an analytical expression for the resonant frequency is then given by

$$
f_{\text {res,lat }}=\frac{1}{2 \pi} \sqrt{\frac{k_{\text {lat }}}{M_{\text {lat }}}},
$$

where

$$
k_{\text {lat }}=\frac{\left(\beta_{i} L\right)^{4} E I_{\text {lat }}}{L^{3}},
$$

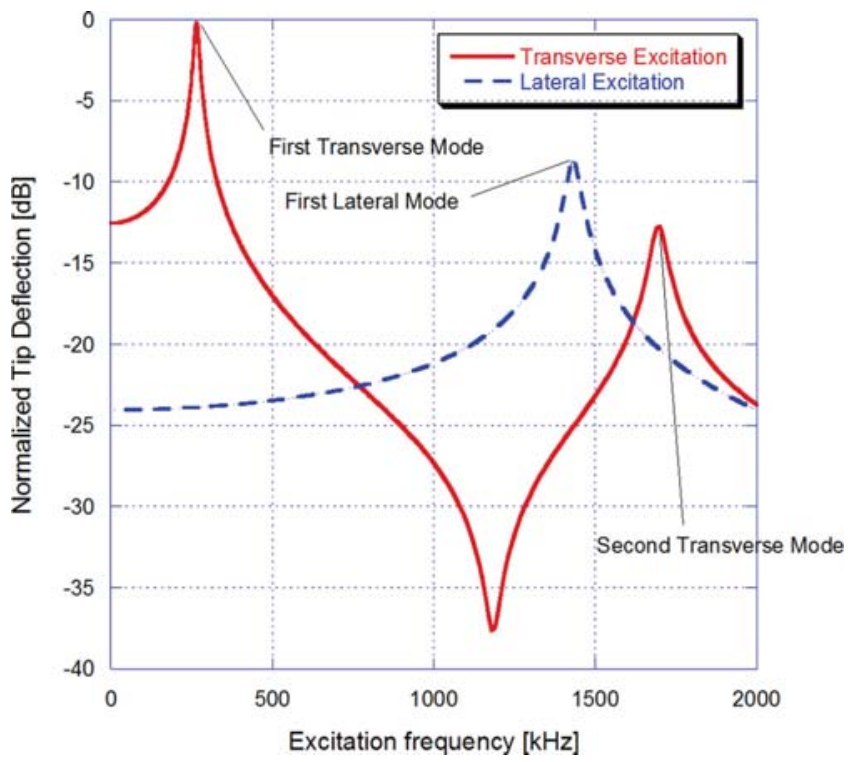

FIG. 6. (Color online) The simulated resonance curve of a $200 \times 45 \times 12 \mu \mathrm{m}^{3}$ silicon microcantilever, assuming a Young's modulus of $169 \mathrm{GPa}$ and a density of $2330 \mathrm{~kg} / \mathrm{m}^{3}$, excited at its free end both in-plane and out-of-plane in water.

$$
\begin{aligned}
& M_{\text {lat }}=\left(\rho_{B} b h L+L g_{2, l a t}\right) \\
& +L \frac{\left(\left(g_{1, \text { lat }} / \omega\right)+\left(\frac{\omega_{\text {res,lat }}}{2}\right) \frac{d}{d \omega}\left(g_{1, \text { lat }} / \omega\right)\right)}{\left(\rho_{B} b h+g_{2, \text { lat }}+\left(\frac{\omega_{\text {res,lat }}}{2}\right) \frac{d}{d \omega}\left(g_{2, \text { lat }}\right)\right)}\left(g_{1, \text { lat } / \omega}\right), \\
& \left(\frac{\omega_{\text {res,lat }}}{2}\right) \frac{d}{d \omega}\left(g_{2, l a t}\right)=\frac{-\sqrt{2} \rho_{L} b^{2}}{8 \sqrt{\operatorname{Re}}}\left(3.08\left(\frac{h}{b}\right)^{0.85}+1\right), \\
& \left(\frac{\omega_{\text {res,lat }}}{2}\right) \frac{d}{d \omega}\left(g_{1, \text { lat }} / \omega\right)=\frac{-\sqrt{2} \rho_{L} b^{2}}{4 \operatorname{Re}}\left(2.56-1.321\left(\frac{h}{b}\right)\right) \\
& -\frac{\sqrt{2} \rho_{L} b^{2}}{8 \sqrt{\operatorname{Re}}}\left(3.108\left(\frac{h}{b}\right)^{0.85}+1\right) \text {. }
\end{aligned}
$$

It is noted that the Reynolds number is dependent on the frequency of excitation, $\omega(=2 \pi f)$. Thus, Eq. (15) is a transcendental equation in $\omega$, whose solution for the system's resonant frequency will be obtained by the following process:

$$
f_{\text {res,lat }}=\lim _{n->\infty} F_{1}^{n}\left(f_{\text {guess }}\right),
$$

where $F_{l}{ }^{n}\left(f_{\text {guess }}\right)$ is the function $F_{l}$ applied $n$ times to $f_{\text {guess }}$, $F_{1}\left(f_{\text {guess }}\right)$ is the right-hand side of Eq. (15), and $f_{\text {guess }}$ is a guess value of the resonant frequency. A good initial guess for the resonant frequency could be taken as the resonant frequency of the microcantilever in a vacuum, $f_{0}$, which is given by ${ }^{23}$

$$
f_{0}=\frac{\left(\beta_{i} L\right)^{2} b}{2 \pi L^{2}} \sqrt{\frac{E}{12 \rho_{B}}} .
$$

If $\operatorname{Re} \gg 1$, Eqs. (15c) and (15d) can be considered approximately zero. Equation (15) can then be simplified to

$$
f_{\text {res,lat }}=\frac{\left(\beta_{i} L\right)^{2}}{2 \pi L^{2}} \sqrt{\frac{E I_{\text {lat }}}{\left(\rho_{B} b h+g_{2, \text { lat }}\right)+\left(g_{1, \text { lat }} / \omega\right)^{2}\left(\rho_{B} b h+g_{2, \text { lat }}\right)^{-1}}} .
$$

Equation (18) shows that, as expected, as the beam becomes stiffer, the resonant frequency will increase, and when the total mass or damping increases, the resonant frequency will decrease.

The resonant frequency of laterally vibrating microcantilevers predicted from theory can be compared to the experimentally determined resonant frequencies of laterally vibrating microcantilevers of various geometries given in Ref. 30. An average beam thickness of $14.48 \mu \mathrm{m}$ was reported in Ref. 30. The beam was primarily made of silicon with a nominal Si thickness of $12 \mu \mathrm{m}$. However, there is an additional triple-layer passivation sandwich consisting of alternating plasma-enhanced chemical vapor-deposited oxide and nitride films in order to mitigate the effects of pinholes on the circuitry used to excite the microcantilever. ${ }^{30}$ 
The beam density was assumed to be that of silicon, or $2330 \mathrm{~kg} / \mathrm{m}^{3}$. The Young's modulus of the composite system is a function of the Young's modulus of the individual layers and can be determined from the experimental data. Using the reported resonant frequencies of each beam in air, ${ }^{30}$ an effective Young's modulus appropriate to the composite system was determined. The mass density and dynamic viscosity of air at room temperature can be estimated as $\rho_{\text {air }}=1.205 \mathrm{~kg} / \mathrm{m}^{3}$ and $\eta_{\text {air }}=0.01827 \mathrm{cP}^{64}$ Using these values in Eq. (15), the Young's modulus can be varied until Eq. (15) matches the reported resonant frequency in air of a particular beam. The average effective Young's modulus of the microcantilevers in air calculated using this procedure was $127.5 \mathrm{GPa}$.

Figure 7 shows the comparison of the simulated resonant frequencies to that of the experimentally determined resonant frequencies in air. The lengths of the beams used in Ref. 30 are $200,400,600,800$, and $1000 \mu \mathrm{m}$ and the widths used are $45,60,75$, and $90 \mu \mathrm{m}$. Note the resonant frequency's linear dependence on $b / L^{2}$ when $b / L^{2}<0.001 \mu \mathrm{m}^{-1}$. For shorter beams $(L=200 \mu \mathrm{m})$, the shear deformation, rotational inertia, and support compliance effects, which are not accounted for when applying Euler-Bernoulli beam theory to a perfectly clamped cantilever, ${ }^{65,66}$ become significant, and the resonant frequency's linear dependency on $b / L^{2}$ no longer holds. The effective Young's modulus calculated for these short beams deviates greatly from the average effective Young's modulus used in the simulations $(127.5 \mathrm{GPa})$, which causes the large difference (up to $31.5 \%$ ) between the predicted and the experimentally determined resonant frequencies for the $L=200 \mu \mathrm{m}$ data set. When $b / L^{2}<0.001 \mu \mathrm{m}^{-1}$, the maximum difference drops to $9.8 \%$.

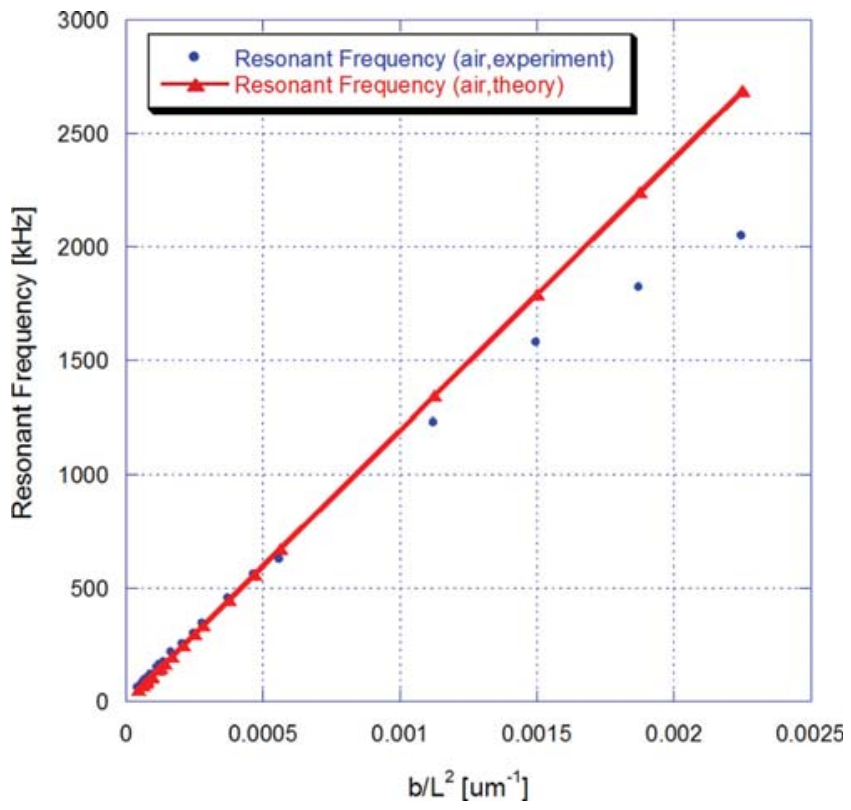

FIG. 7. (Color online) The simulated resonant frequency of laterally excited microcantilevers in air compared to the experimentally determined resonant frequency as a function of $b / L^{2}$ for widths of $45,60,75$, and $90 \mu \mathrm{m}$; lengths of $200,400,600,800$, and $1000 \mu \mathrm{m}$; and a thickness of $14.48 \mu \mathrm{m}$. The Young's modulus of the beam was specified as $127.5 \mathrm{GPa}$, the average effective Young's modulus of the specimens. Experimental data are from Ref. 30.
When the beam is vibrating laterally in a viscous liquid medium, the same qualitative $b / L^{2}$ dependence remains, as was observed in air (see Fig. 8). However, the addition of pressure effects causes a dependence on the thickness. It is noted from Eq. (15) that, while the spring constant $k_{\text {lat }}$ in water is still linearly dependent on the thickness, the thickness dependence of the effective mass $\left(M_{\text {lat }}\right)$ is not straightforward and depends on the ratio of the beam mass to effective displaced fluid mass. As $h$ increases and exceeds $b$, the resonant frequency reduces to that of a microcantilever undergoing out-of-plane vibration with width $h$ and thickness $b$. The theory again diverges from the experimental data when the beam is shorter, i.e., for $b / L^{2}>0.001 \mu \mathrm{m}^{-1}$. The maximum percentage difference between the simulated resonant frequency and the experimentally determined resonant frequency in water is $28.7 \%$. When $b / L^{2}<0.001 \mu \mathrm{m}^{-1}$, this difference drops to $11.8 \%$.

The resonant frequency can also be investigated as a function of the properties of the liquid medium. The resonant frequency of a laterally vibrating beam decreases from its value in air, $f_{0},{ }^{3}$ by

$$
\Delta f=f_{0}\left(1-\sqrt{\frac{\rho_{B} L b h}{M_{l a t}}}\right) .
$$

As the viscosity or density of the medium is increased, the effective mass, $M_{\text {lat }}$, will increase. The theoretical results were compared to the results from experiments performed in different percent aqueous glycerol mixtures, providing a range of dynamic viscosities and densities for the liquid medium. A microcantilever with a geometry of $200 \times 60 \times 6.7 \mu \mathrm{m}^{3}$, fabricated using the same method as in Ref. 30, was used in the experiments with aqueous glycerol solutions (up to $20 \% \mathrm{w} / \mathrm{w}$,

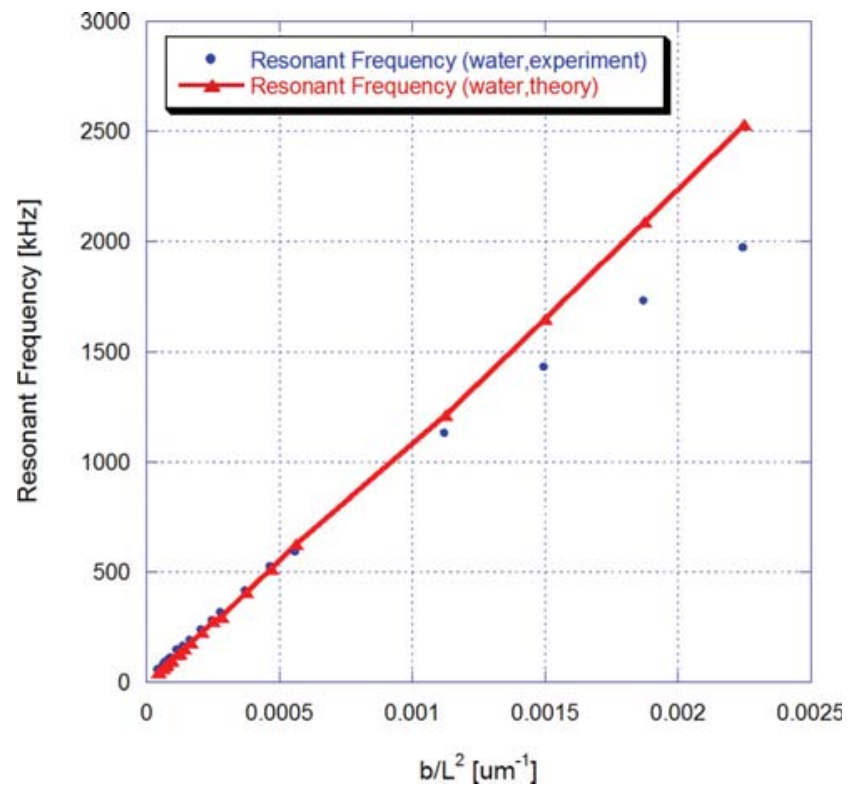

FIG. 8. (Color online) The simulated resonant frequency of laterally excited microcantilevers in water compared to the experimentally determined resonant frequency as a function of $b / L^{2}$ for widths of $45,60,75$, and $90 \mu \mathrm{m}$; lengths of 200, 400,600,800, and $1000 \mu \mathrm{m}$; and a thickness of $14.48 \mu \mathrm{m}$. The Young's modulus of the beam was assumed to be $127.5 \mathrm{GPa}$. Experimental data comes from Ref. 30. 

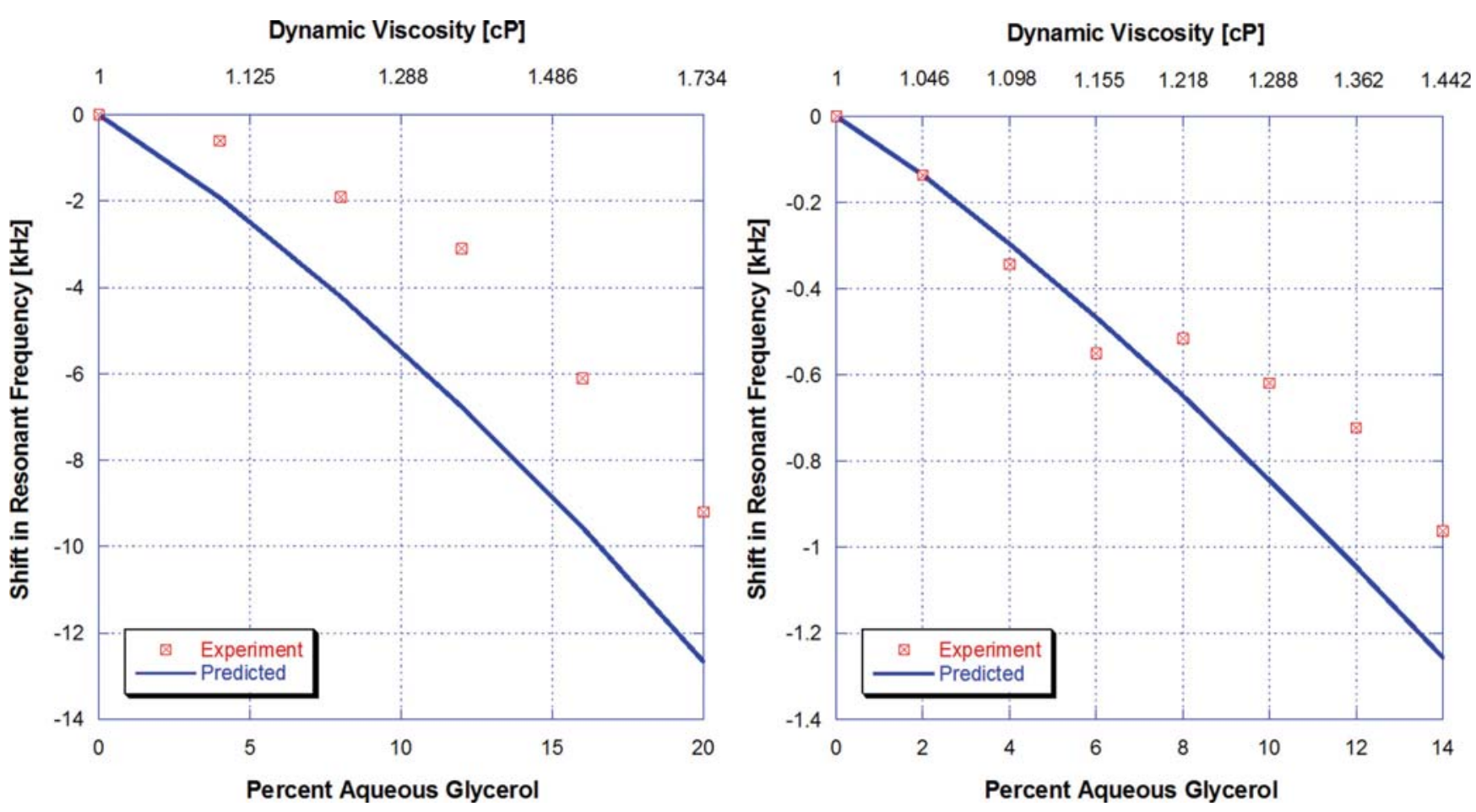

FIG. 9. (Color online) (a) and (b): Predicted and experimentally determined shift in the resonant frequency of laterally vibrating microcantilever from water to a solution of aqueous glycerol with a geometry of (a) $200 \times 60 \times 6.7 \mu \mathrm{m}^{3}$ and (b) $1000 \times 90 \times 10.9 \mu \mathrm{m}^{3}$.

or $1.734 \mathrm{cP}$ ). The shift in the resonant frequency from its value in water as a function of percent aqueous glycerol is given in Fig. 9(a). The predicted shift in the resonant frequency using Eq. (19) is also shown. Using Eq. (17) and the experimentally determined resonant frequency in air, the effective Young's modulus for this beam was determined to be $85.4 \mathrm{GPa}$. This value is smaller due to the model not incorporating the shear deformation, rotational inertia, and support compliance effects of the short beam. The theoretical results calculated using Eq. (19) show similar trends; however, the theory overestimates the frequency drop. When using longer beams (i.e., $1000 \times 90 \times 10.9 \mu \mathrm{m}^{3}$ ), the predicted decrease in the resonant frequency has shown better agreement with the experimental results (see Fig. 9(b)). This suggests that the difference could be due to effects that are not accounted for when the assumption of a long beam is made. This assumption neglects the effects of the shear and rotational inertia of the beam, which cause the resonant frequency to be lower. These effects tend to be larger for shorter beams, which have higher quality factors compared to longer beams.

It can be seen from Fig. 6 that care should be taken when choosing the beam's geometry so that the lateral flexural mode's resonant frequency does not interfere with the resonant frequency of a higher-order transverse flexural mode, which would cause interaction between the two modes, especially when using the device in an oscillator circuit in various applications. Using Eq. (15), the aspect ratio at which two modes have the same resonant frequency and could thus interfere with each other is

$$
\frac{h}{b}=\left(\frac{\beta_{i}}{\beta_{j}}\right)^{2} \sqrt{\frac{M_{\text {trans }}}{M_{\text {lat }}}},
$$

where $i$ and $j$ are the mode numbers of the particular lateral and transverse flexural modes, respectively, that will cause interference, and $M_{\text {trans }}$ is calculated using Eq. (15b) and the well-known values for $g_{1}$ and $g_{2}$ of a transversely vibrating beam instead of $g_{1, \text { lat }}$ and $g_{2, \text { lat }}$.

From Eqs. (15) and (20), it can be seen that vibrating microcantilevers in the in-plane direction as opposed to the out-of-plane direction increases the resonant frequency of the same mode number by a factor of

$$
\frac{f_{\text {res }, \text { lat }, i}}{f_{\text {res }, \text { trans }, i}}=\frac{b}{h} \sqrt{\frac{M_{\text {trans }}}{M_{\text {lat }}}} .
$$

When operating in vacuum or a low density medium, such as air, the effective masses are the same (essentially just the beam mass) and the resonant frequency increases by a factor of $b / h$. In a viscous and/or high-density liquid medium, the ratio of the effective masses is greater than one and the ratio of the resonant frequencies is, therefore, larger than $b / h$. For example, for the beams investigated in Ref. 30, the fundamental resonant frequency of transversely excited beams is predicted to decrease by $32 \%$ to $49 \%$ when placed in water. However, the resonant frequency of the same beams vibrating laterally is predicted to drop by only $5.8 \%$ to $15 \%$. For comparison, the experimental data in Ref. 30 shows a drop of $3.7 \%$ to $18.3 \%$ for the beams undergoing lateral excitation. The ratio of the resonant frequencies of laterally and transversely vibrating beams is thus higher in water (ranging from 4.5 to 12.12 for the geometries given in Ref. 30) compared to the ratios when operating in air (ranging from 3.31 to 6.62 , or roughly $b / h$ ). This is because the difference in the effective masses (beam mass plus effective fluid mass) increases as the dynamic viscosity and density of the 
medium increase. As this trend continues for higher viscosities, it is thus even more advantageous to operate in the in-plane flexural mode when operating in high-viscosity media in chemical (or biological) sensor applications.

\section{Quality factor}

A fundamental problem encountered by microcantilevers operating in the liquid phase is the drastic decrease in the microcantilever's quality factor, denoted $Q$, compared to the corresponding quality factor in the gas phase. This decrease in the quality factor increases the frequency noise (which is proportional to $f_{\text {res }} / Q$ when operating in an oscillator configuration ${ }^{40,67,68}$ ), thus increasing the limit of detection (LOD) in biochemical sensing applications. The quality factor is defined as $2 \pi$ times the ratio of the maximum energy stored in a resonating system to the amount of energy dissipated in one cycle. ${ }^{69}$ The $2 \pi$ keeps the definition consistent with a second definition, which is the ratio of the resonant frequency to the $3-\mathrm{dB}$ bandwidth of the system,

$$
Q_{\text {lat }, 3 d B}=f_{\text {res, lat }} / \Delta f_{3 d B} .
$$

It is common when working with microcantilever systems to use the 3 -dB bandwidth definition, ${ }^{23,32,68,70}$ and it is noted that, when the loss is low, the two definitions are equivalent.

If it is assumed that $g_{1, \text { lat }}$ and $g_{2, \text { lat }}$ are frequencyindependent within the 3-dB bandwidth, an expression for the quality factor can be derived from the equation of motion as

$$
Q_{\text {lat }}=\left(2\left(1-\sqrt{1-\frac{g_{1, \text { lat }} / \omega}{\rho_{B} b h+g_{2, \text { lat }}}}\right)\right)^{-1} .
$$

When Re $\gg 1$, using Eqs. (3) and (4), Eq. (23) reduces to

$$
Q_{\text {approx }}=\frac{\rho_{B} b h+g_{2, l a t}}{g_{1, \text { lat }} / \omega},
$$

which is identical to the expression for the quality factor published in the literature when low loss is assumed. ${ }^{23,61}$

If it is assumed that the beam's mass is much larger than the effective fluid mass, Eq. (24) can be rewritten as

$$
Q_{\text {approx }}=\frac{\rho_{B} h \sqrt{2 \pi f_{\text {res,lat }}}}{\sqrt{2 \eta \rho_{L}} C_{I}}, \quad\left(\rho_{B} h b \gg g_{2, \text { lat }}\right) .
$$

Equation (25) will underestimate the quality factor, since it neglects the energy associated with the effective fluid mass. Even though $f_{\text {res,lat }}$ is approximately dependent on $\rho_{B}{ }^{-1 / 2}$, it can still be seen from Eq. (25) that the quality factor is improved when increasing the density of the beam material. This makes sense because, for a given vibration amplitude, increasing the density of the beam increases the amount of kinetic energy (converted to stored energy during a cycle of vibration), while keeping the amount of fluidic damping constant. Neglecting viscosity's effect on $C_{I}$, Eq. (25) also shows, contrary to intuition, that the majority of the drop in the system's quality factor when operating in water as opposed to air arises from the increase in the medium's density $\left(1.205\right.$ to $998.23 \mathrm{~kg} / \mathrm{m}^{3}$ for $\left.20{ }^{\circ} \mathrm{C}\right)$ instead of the increase in the medium's viscosity $(0.01827$ to $1 \mathrm{cP}$ for $\left.20{ }^{\circ} \mathrm{C}\right) .{ }^{64}$ Using the relationship of the resonant frequency to the beam geometry given in Sec. IV B, the quality factor is found to be a linear function of $h b^{1 / 2} / L$, implying that shorter, thicker and wider beams should have higher quality factors. This result is consistent with the result derived earlier in Refs. 49 and 50 by a different method.

The quality factor was analyzed using both the numerical results obtained using FEA and the approximate fit of the hydrodynamic function. As noted previously, the largest discrepancy between the numerical results and the approximate fit given by Eq. (11) is when the Reynolds number is large. It is then expected that the geometries investigated with the largest Reynolds numbers would also produce the largest discrepancy in the quality factor. For the range of geometries investigated in this work, the largest Reynolds numbers are found when $L=200 \mu \mathrm{m}$. The calculated quality factors were found to be within $5 \%$ of those obtained using the results from the finite element model. It is then assumed that Eq. (11) is appropriate to use when calculating characteristics of laterally vibrating microcantilevers in liquid phase.

The predicted quality factors calculated using Eq. (23) were compared to those experimentally determined in water as a function of $b^{1 / 2} / L$ in Fig. 10. The simulation assumed a laterally vibrating beam in water with a Young's modulus of 127.5 GPa and a beam density of $2330 \mathrm{~kg} / \mathrm{m}^{3}$. While the predicted quality factors ranged from $22.3 \%$ lower to $36.2 \%$ higher than the experimentally determined quality factors given by Ref. 30, the trend was found to still follow the $b^{1 / 2} / L$ dependency. This confirms that shorter and wider beams have higher quality factors. The discontinuities in Fig. 10 are due to changes in the beam's length. Thicker beams are also expected to

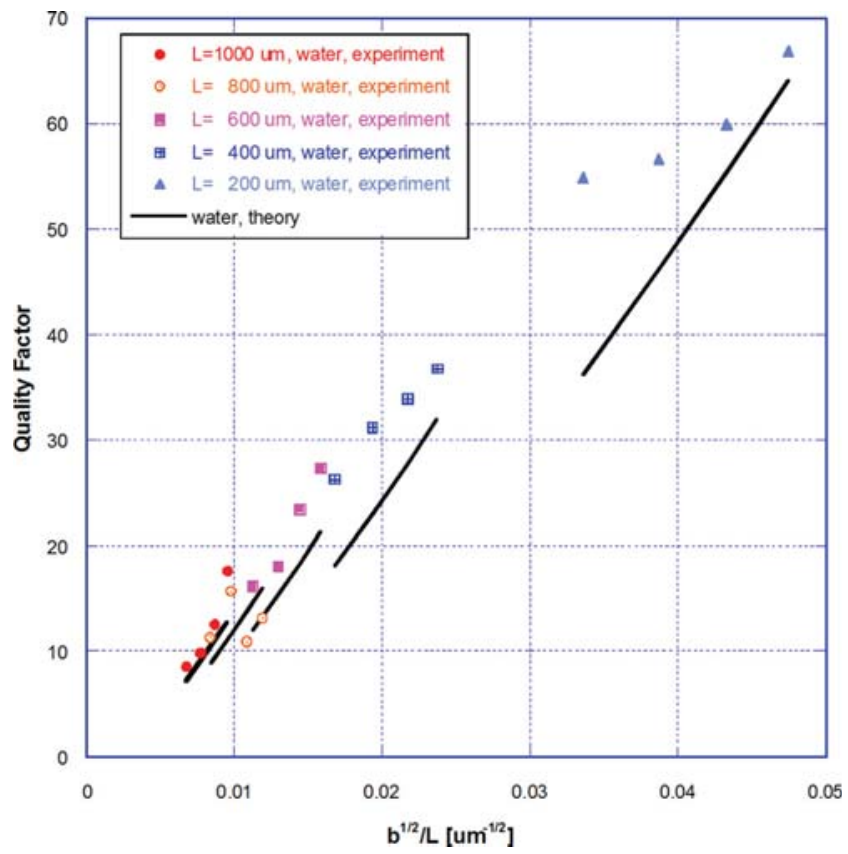

FIG. 10. (Color online) Quality factors of laterally vibrating microcantilevers in water. The width is varied between 45 and $90 \mu \mathrm{m}$, the length from 200 to $1000 \mu \mathrm{m}$, and the thickness is set at $14.48 \mu \mathrm{m}$. The Young's modulus of the beam was assumed as $127.5 \mathrm{GPa}$. 
increase the quality factor by increasing the mass and stored energy of the beam; however, this trend does not hold as $h / b$ approaches 1 . Figure 11 shows this effect, both experimentally and theoretically, for a beam of $200 \mu \mathrm{m}$ length and $60 \mu \mathrm{m}$ width. A Young's modulus of $127.5 \mathrm{GPa}$ was assumed for all thicknesses. Note the roughly linear increase in both the predicted quality factor and the experimentally determined quality factor. Also note that the slope of this increase and, thus, the benefit of using thicker beams decreases as the thickness increases.

The quality factor will also change as a function of the medium of operation. Figure 12 shows the quality factor of a $200 \times 60 \times 6.7 \mu \mathrm{m}^{3}$ beam as a function of percent aqueous glycerol. The theoretically predicted quality factors calculated using Eq. (23) are also given. While both the experimental results and the theoretically predicted results show similar trends, the experimentally determined quality factors are again larger than the theoretically predicted quality factors. Calculated values for the quality factors using only Stokes fluid resistance (using Eq. (9) for the hydrodynamic function) are also shown in Fig. 12. However, this expression neglects the pressure effects of the fluid on the smaller faces (of dimension $h$ ), i.e., it is based on assuming only a Stokestype fluid resistance on the top and bottom faces of the beam. The theoretically predicted quality factors would be smaller if the shear deformation and support compliance effects were taken into account. Thus, these effects are not the source of the discrepancy. Another assumption made when calculating the hydrodynamic forces was that the beam was infinitely long. This assumption was made to neglect the edge effects near the end of the beam in the lengthwise direction and might not be appropriate for the given geometry. It is noted that the quality factors of longer beams (e.g.,

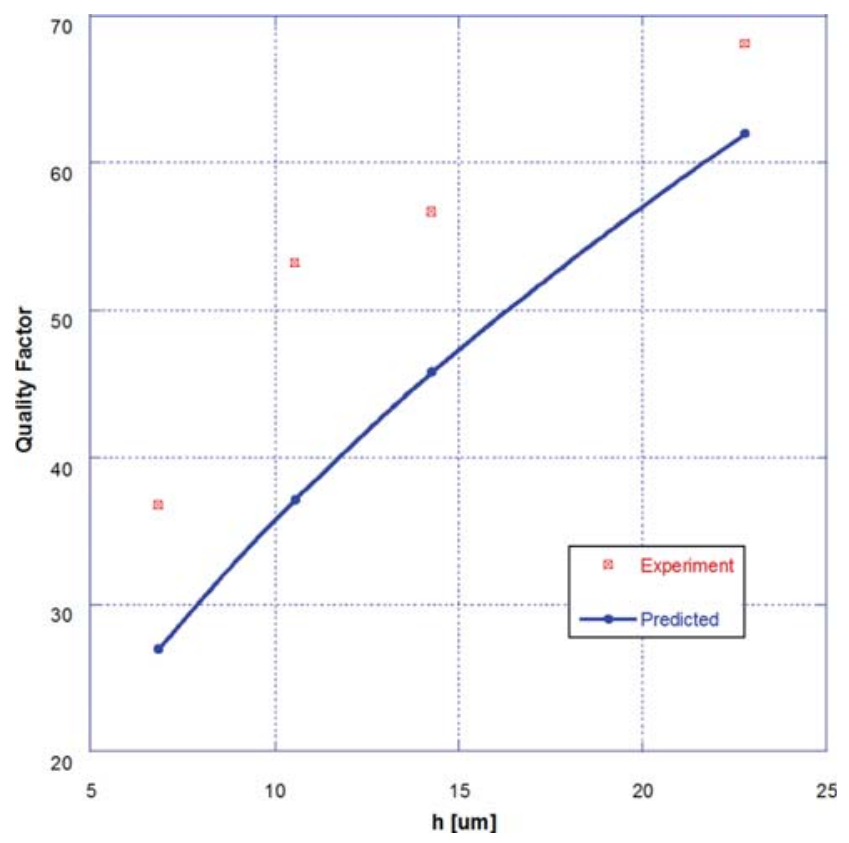

FIG. 11. (Color online) Quality factors of laterally vibrating microcantilevers in water with lengths of $200 \mu \mathrm{m}$, widths of $60 \mu \mathrm{m}$, and varying thickness from $6.83 \mu \mathrm{m}$ to $22.77 \mu \mathrm{m}$. The Young's modulus of the beam was assumed as $127.5 \mathrm{GPa}$. Experimental data comes from Ref. 30 .

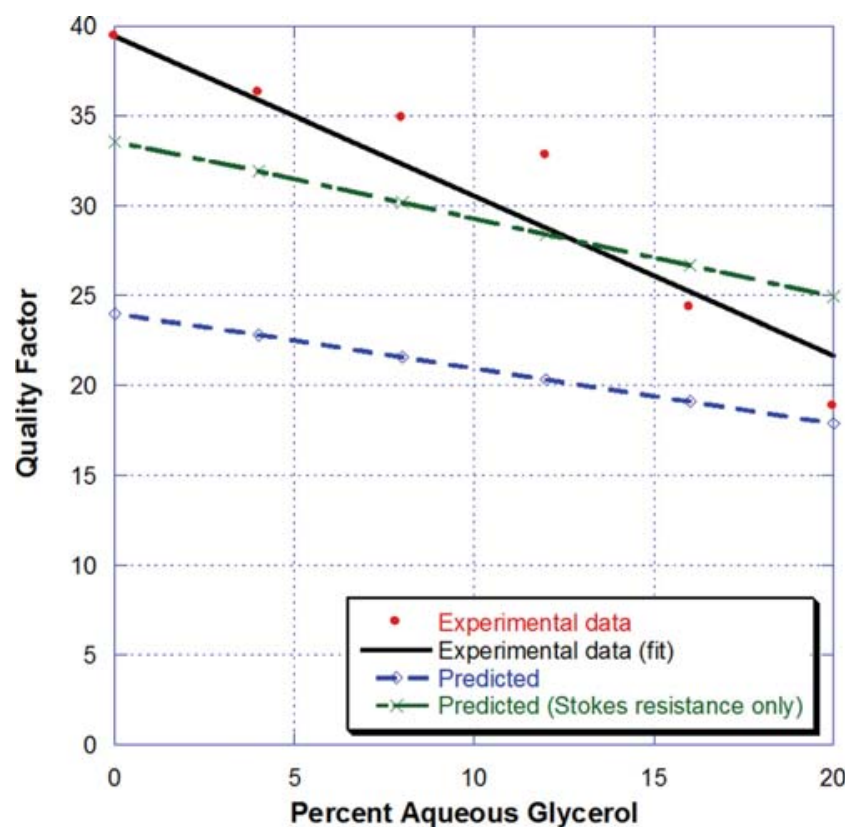

FIG. 12. (Color online) Predicted and experimentally determined quality factors of a $200 \times 60 \times 6.7 \mu \mathrm{m}^{3}$ laterally vibrating microcantilever as a function of percent aqueous glycerol.

$1000 \times 90 \times 10.9 \mu \mathrm{m}^{3}$ ), not shown here, more closely match the characteristics predicted by theory, just as with the resonant frequency.

The experimentally determined quality factors are larger than the commonly obtained values for transversely vibrating beams in water, which are usually on the order of $10 .^{24}$ Even with the addition of the thickness and edge effects, the quality factor of a laterally vibrating beam is still higher than that of the same beam vibrating transversely. Using Eq. 25, the ratio of the quality factor of a laterally vibrating beam to that of a transversely vibrating beam of similar geometry is approximately given by

$$
\frac{Q_{\text {lat,approx }}}{Q_{\text {trans,approx }}}=\frac{\rho_{B} b h+g_{2, \text { lat }}}{\rho_{B} b h+g_{2, \text { trans }}} \frac{\Gamma_{I, \text { trans }}}{\Gamma_{I, \text { lat }}} .
$$

Note that the first fraction on the right-hand side of Eq. (26) is smaller than one (when $b>h$ ), as transversely vibrating beams yield a larger effective fluid mass compared to laterally vibrating beams. The second fraction is the ratio of the amounts of viscous damping, which is normally much greater than one for the cases considered. The improvement in the quality factor is also a function of the medium of operation. Unlike the comparable trend in the resonant frequency, the improvement is smaller for media with higher viscosities and densities. The predicted improvement in the quality factor for the beams investigated in Ref. 30 ranges from a factor of 3 to 4.5 in air and from a factor of 1.55 to 2.53 in water. Since the experimentally determined quality factors are found to be higher than the predicted quality factors in practice, the improvements will be larger. However, the best improvements in the predicted quality factor are obtained for the shortest and widest beams investigated. Thus, shorter and wider beams have both larger resonant frequencies (and thus 
higher sensitivities in chemical sensor applications) and higher quality factors (and thus better limits of detection).

\section{CONCLUSIONS}

The resonant frequency and quality factor of microcantilevers vibrating in the in-plane direction in a viscous liquid medium are analyzed in terms of the beam's geometry and the properties of the medium of operation and compared to those of microcantilevers vibrating in the out-of-plane direction. The hydrodynamic force acting on the beam is evaluated, accounting for both the shear force and the pressure force. It is noted that, even with relatively thin beams, there is a need to account for the effects of thickness to obtain accurate estimates of the hydrodynamic forces. A set of correction factors are determined for modifying the hydrodynamic function given by Stokes for an infinitely wide beam to include both the edge effects and the effects of the pressure acting on the thickness.

Theoretical results show that the resonant frequency of beams vibrating in the in-plane direction will be at least a factor of $b / h$ higher than that of beams vibrating in the outof-plane direction. The ratio of the resonant frequencies will be higher for beams operating in media with higher densities and dynamic viscosities. The calculated resonant frequencies are shown to match trends in the experimentally determined resonant frequencies published in the literature, provided that the beam is long enough to neglect shear deformation, rotational inertia, and support compliance effects. The quality factor is shown to be a function of beam density, fluid density, fluid viscosity, and $h b^{1 / 2} / L$. Shorter, thicker, and wider beams vibrating laterally are shown to produce higher quality factors compared to longer beams of a smaller cross section. It is shown from experiments with viscous solutions that laterally vibrating microcantilevers can be excited and have relatively high (20 to 60) quality factors, even when operating in media with higher dynamic viscosities than water. When compared to transversely vibrating beams, the predicted characteristics (i.e., the resonant frequency, quality factor) are shown to be higher. For chemical sensor applications, considering the resonant frequency and mass sensitivity, this increase is larger for media with higher dynamic viscosities.

\section{ACKNOWLEDGMENTS}

This work is supported in part by NSF Grant No. ECCS0824017 and by the French National Agency (ANR) (I. Dufour) (MicRheo project $\mathrm{n}^{\circ}$ ANR-08-NANO-004). The authors would like to thank L. Beardslee for the experimental data referred to in the work, Jinjin Zhang for the viscosity measurements, and Tao Cai for helpful discussion.

\footnotetext{
${ }^{1}$ M. Sepaniak, P. Datskos, N. Lavrik, and C. Tipple, Anal. Chem. 74, 21 (2002).

${ }^{2}$ K. Goeders, J. Colton, and L. Bottomley, Chem. Rev. 108, 2 (2008).

${ }^{3}$ D. Lange, C. Hagleitner, A. Hierlemann, O. Brand, and H. Baltes, Anal. Chem. 74, 3084 (2002)

${ }^{4}$ C. Vančura, M. Ruegg, Y. Li, D. Lange, C. Hagleitner, O. Brand, A. Hierlemann, and H. Baltes, in Transducers 2003, 12th International Conference on Solid-State Sensors, Actuators and Microsystems, Boston, MA, 8-12 June 2003 (IEEE, Boston, 2003), Vol. 2, pp 1355-1358.
}

${ }^{5}$ B. Rogers, L. Manning, M. Jones, T. Sulchek, K. Murray, B. Beneschott, J. D. Adams, Z. Hu, T. Thundat, H. Cavazos, and S. C. Minne, Rev. Sci. Instrum. 74, 11 (2003).

${ }^{6}$ T. Thundat, E. A. Wachter, S. L. Sharp, and S. J. Warmack, Appl. Phys. Lett. 66, 13 (1995).

${ }^{7}$ L. A. Pinnaduwage, H.-F. Ji, and T. Thundat, IEEE Sens. J. 5, 4 (2005).

${ }^{8}$ S. Tétin, B. Caillard, F. Ménil, H. Debéda, C. Lucat, C. Pellet, and I. Dufour, Sens. Actuators B 143, 818 (2010).

${ }^{9}$ P. Kapa, P. Liu, A. Bandhanadham, J. Fang, K. Varahramyan, W. Davis, and H.-F., Ji., Sens. Actuators B 134, 2 (2008).

${ }^{10}$ M. R. Plata, J. Hernando, M. Zougagh, A. M. Contento, M. J. Villasenor, J. L. Sanchez- Rojas, and A. Rios, Sens. Actuators B 134, 1 (2008).

${ }^{11}$ L. A. Pinnaduwage, V. Boiadjiev, J. E. Hawk, and T. Thundat, Appl. Phys. Lett. 83, 7 (2003).

${ }^{12}$ N. Lavrik and P. Datskos, Appl. Phys. Lett. 82, 16 (2003).

${ }^{13}$ B. Ilic, H. G. Craighead, S. Krylov, W. Senaratne, C. Ober, and P. Neuzil, J. Appl. Phys. 95, 3694 (2004).

${ }^{14}$ A. Boisen, J. Thaysen, H. Jensenius, and O. Hansen, Ultramicroscopy 82, 11 (2000).

${ }^{15}$ A. R. Kadam, G. P. Nordin, and M. A. George, J. Appl. Phys. 99, 094905 (2006)

${ }^{16}$ H. Jensenius, J. Thaysen, A. A. Rasmussen, L. H. Veje, O. Hansen, and A. Boisen, Appl. Phys. Lett. 76, 18 (2000).

${ }^{17}$ D. R. Baselt, B. Fruhberger, E. Klaasen, S. Cemalovic, C. L. Britton, Jr., S. V. Patel, T. E. Mlsna, D. McCorkle, and B. Warmack, Sens. Actuators B 88, 2 (2003).

${ }^{18}$ C. W. Van Neste, L. R. Senesac, D. Yi, and T. Thundat, Appl. Phys. Lett. 92, 134102 (2008).

${ }^{19}$ L. A. Pinnaduwage, W. Zhao, A. C. Gehl, S. L. Allman, A. Shepp, K. K. Mahmud, and J. W. Leis, Appl. Phys. Lett. 91, 044105 (2007).

${ }^{20}$ K. Yasumura, T. Stowe, E. Chow, T. Pfafman, T. Kenny, B. Stipe, and D. Rugar, J. Microelectromech. Syst. 9, 1 (2000).

${ }^{21}$ M. Maute, S. Raible, F. E. Prins, D. P. Kern, H. Ulmer, U. Weimar, and W. Gopel, Sens. Actuators B 58, 1 (1999).

${ }^{22}$ I. Dufour, S. Heinrich, and F. Josse, J. Microelectromech. Syst. 16, 1 (2007).

${ }^{23}$ J. Sader, J. Appl. Phys. 84, 1 (1998).

${ }^{24}$ J. Chon, P. Mulvaney, and J. Sader, J. Appl. Phys. 87, 8 (2000)

${ }^{25}$ A. Boisen and T. Thundat, Mater. Today 12, 9 (2009).

${ }^{26}$ P. I. Oden, G. Y. Chen, R. A. Steele, R. J. Warmack, and T. Thundat, Appl. Phys. Lett. 68, 26 (1996).

${ }^{27}$ C. Vančura, I. Dufour, S. Heinrich, F. Josse, and A. Hierlemann, Sens. Actuators, A 141, 1 (2008)

${ }^{28} \mathrm{C}$. Vančura, J. Lichtenberg, A. Hierlemann, and F. Josse, Appl. Phys. Lett. 87, 162510 (2005)

${ }^{29}$ X. Xu and A. Raman, J. Appl. Phys. 102, 034303 (2007).

${ }^{30}$ L. Beardslee, A. M. Addous, S. M. Heinrich, F. Josse, I. Dufour, and O. Brand, J. Microelectromech. Syst. 19, 4 (2010).

${ }^{31}$ S. Basak and A. Raman, J. Appl. Phys. 99, 114906 (2006).

${ }^{32}$ R. Cox, F. Josse, M. Wenzel, S. Heinrich, and I. Dufour, Anal. Chem. 80, 15 (2008).

${ }^{33}$ F. Lochon, I. Dufour, and D. Rebiere, Sens. Actuators B 108, 1 (2005).

${ }^{34}$ K. Naeli and O. Brand, J. Appl. Phys. 105, 014908 (2009).

${ }^{35}$ L. B. Sharos, A. Raman, S. Crittenden, and R. Reifenberger, Appl. Phys. Lett. 84, 23 (2004)

${ }^{36}$ C. A. Van Eysden and J. Sader, J. Appl. Phys. 101, 044908 (2007).

${ }^{37}$ X. Xia, P. Zhou, and X. Li, Proc. IEEE Sens. 2008, 577 (2008).

${ }^{38}$ I. Bargatin, I. Kozinsky, and M. L. Roukes, Appl. Phys. Lett. 90, 093116 (2007).

${ }^{39}$ Z. Hao, A. Erbil, and F. Ayazi, Sens. Actuators, A 109, 156 (2003).

${ }^{40}$ C. Vančura, Y. Li, J. Lichtenberg, K.-U. Kirstein, A. Hierlemann, and F. Josse, Anal. Chem. 79, 4 (2007).

${ }^{41}$ D. Paci, K. U. Kirstein, C. Vančura, J. Lichtenberg, and H. Baltes, Analog Integr. Circuits Signal Process. 44, 119 (2005).

${ }^{42}$ C. Hagleitner, D. Lange, A. Hierlemann, O. Brand, and H. Baltes, IEEE J. Solid-State Circuits 37, 12 (2002).

${ }^{43}$ J. Pei, F. Tian, and T. Thundat, Anal. Chem. 76, 2 (2004)

${ }^{44}$ M. Calleja, J. Tamayo, A. Johansson, P. Rasmussen, L. Lechuga, and A. Boisen, Sens. Lett. 1, 1 (2003).

${ }^{45}$ N. V. Lavrik, M. J. Sepaniak, and P. G. Datskos, Rev. Sci. Instrum. 75, 7 (2004).

${ }^{46}$ I. Dufour and F. Ludivine, Sens. Actuators B 91, 353 (2003).

${ }^{47}$ D. Brumley, M. Willcox, and J. Sader, Phys. Fluids 22, 052001 (2010). 
${ }^{48} \mathrm{X}$. Zhang and W. Tang, in Proceedings of IEEE Workshop on Micro Electro Mechanical Systems, Oiso, Japan, 25-28 January 1994 (MEMS '94, Proceedings, 1994), pp 199-204.

${ }^{49}$ S. M. Heinrich, R. Maharjan, L. Beardslee, O. Brand, I. Dufour, and F. Josse, in Proceedings of the International Workshop on Nanomechanical Cantilever Sensors, Banff, Canada, 26-28 May 2010.

${ }^{50}$ S. M. Heinrich, R. Maharjan, I. Dufour, F. Josse, L. Beardslee, and O. Brand, in Proceedings of the IEEE Sensors 2010 Conference, Waikoloa, HI, 1-4 November 2010.

${ }^{51}$ R. Cox, F. Josse, S. M. Heinrich, I. Dufour, and O. Brand, in Proceedings of the IEEE International Frequency Control Symposium, Newport Beach, CA, 2-4 June 2010

${ }^{52}$ E. Tuck, J. Eng. Math. 3, 1 (1969).

${ }^{53}$ R. P. Kanwal, Q. J. Mech. Appl. Math. 8, 2 (1955).

${ }^{54}$ C. A. Eysden and J. Sader, Phys. Fluids 18, 123102 (2006).

${ }^{55}$ R. Clarke, S. Cox, P. Williams, and O. Jensen, J. Fluid Mech. 545, 397 (2005).

${ }^{56}$ G. Stokes, Trans. Cambridge Philos. Soc. 9, 6 (1851)

${ }^{57}$ S. P. Hu, J. Mech. 21, 1 (2005).

${ }^{58}$ W. Zhang and K. Turner, Sens. Actuators, A 134, 2 (2007).
${ }^{59}$ T. Iyengar, N. Srinivasacharyulu, and J. Ramana, J. Appl. Math. Phys. 39, 649 (1988)

${ }^{60}$ H. Hosaka, K. Itao, and S. Kuroda, Sens. Actuators A 49, 87 (1995).

${ }^{61}$ A. Maali, C. Hurth, R. Boisgard, C. Jai, T. Cohen-Bouhacina, and J. Aimé, J. Appl. Phys. 97, 074907 (2005).

${ }^{62}$ Laminar Boundary Layers, edited by L. Rosenhead (Clarendon, Oxford, 1963).

${ }^{63}$ M. Hopcroft, W. Nix, and T. Kenny, J. Microelectromech. Syst. 19, 2 (2010).

${ }^{64}$ Handbook of Chemistry and Physics, edited by R. Weast (CRC, Cleveland, 1973).

${ }^{65}$ S. P. Timoshenko, Philos. Mag. 6, 125 (1921).

${ }^{66}$ T. C. Huang, J. Appl. Mech. 28, 579 (1961).

${ }^{67}$ I. Dufour, F. Lochon, S. Heinrich, F. Josse, and D. Rebière, IEEE Sens. J. 7, 44 (2007).

${ }^{68}$ L. Fadel, I. Dufour, F. Lochon, and O. Francais, Sens. Actuators B 102, 73 (2004).

${ }^{69}$ B. Razavi, IEEE J. Solid-State Circuits 31, 3 (1996).

${ }^{70}$ T. Thundat, R. J. Warmack, G. Y. Chen, and D. P. Allison, Appl. Phys. Lett. 64, 1563 (1994) 